\title{
POSSIBILITIES OF MEASURING HYDROGEN IN NATURAL GAS
}

\author{
Ildikó Bölkény \\ research assistant, University of Miskolc, Research Institute of Electronics and Informatics \\ 3515 Miskolc, Miskolc-Egyetemváros, e-mail: bolkeny@eiki.hu \\ Gábor Hegyi \\ research assistant, University of Miskolc, Research Institute of Electronics and Informatics \\ 3515 Miskolc, Miskolc-Egyetemváros, e-mail: eikhega@uni-miskolc.hu \\ Angéla Váradiné Szarka \\ institute director, University of Miskolc, Research Institute of Electronics and Informatics \\ 3515 Miskolc, Miskolc-Egyetemváros, e-mail: angela.varadi@eiki.hu
}

\begin{abstract}
Green hydrogen produced by renewable energies is an innovative use of mixed with natural gas to deliver the mixture to end users using existing natural gas storage and networks, thereby achieving increased performance. In such a new technology, measuring the hydrogen content of a hydrogen natural gas mixture is essential. This paper provides an overview of hydrogen sensors.
\end{abstract}

Keywords: hydrogen, natural gas-hydrogen mixture, hydrogen sensors

\section{Introduction}

The use of hydrogen as an energy source seems to be spreading more and more, as its combustion processes only produce water vapor, but not carbon dioxide. Green hydrogen produced by renewable energies is an innovative use of mixed with natural gas to deliver the mixture to end users using existing natural gas storage and networks, thereby achieving increased performance. Natural gas is one of the most important primary energy sources to date. With the help of green hydrogen, natural gas can be made more efficient and environmentally friendly, especially when used on an existing technology.

Pressure, temperature and flow transmitters used in the oil and gas industry have been operating on the natural gas network for years. However, with different ratios of hydrogen, it becomes necessary to measure the concentration of hydrogen as well. The following section introduces hydrogen sensors that are suitable for measuring hydrogen in gas. Then two industry solutions will be presented.

\section{The hydrogen sensors}

The catalytic sensors. This sensors work based on combustible gases. The basic principle is that these gases react with the catalytic surface of the sensor and creates heat. (Hübert et al., 2011) This can then be measured. Hydrogen is highly combustible and has a standard heat of combustion around 141,9 $\mathrm{kJ} / \mathrm{g}$, which compared to natural gas (around $42-55 \mathrm{~kJ} / \mathrm{g}$ (WNA, 2021)) is around three times higher. This means that hydrogen can be measured in natural gas with catalytic sensors. (Hübert et al., 2011)

The first type of catalytic sensor is the pellistor. In pellistors there are two platinum coils embedded in a porous pellet. One of these pellets is an active bead, which means that, its surface is plated with a catalytic material, such as palladium or platinum. The other pellet, the inactive bead, is used to com- 
pensate the ambient parameters, such as humidity. These beads function as heater elements and resistive thermometers. The basic principle for the sensor type is the ambient gas has the same effect on both pellets, so the inactive bead compensates its effects. If combustible gas is introduced, it will react with the surface of the active pellet and will create heat, which creates a resistive difference between the beads. This difference can be measured by a Wheatstone bridge. (Hübert et al., 2011) The pellistor is shown in Figure 1.

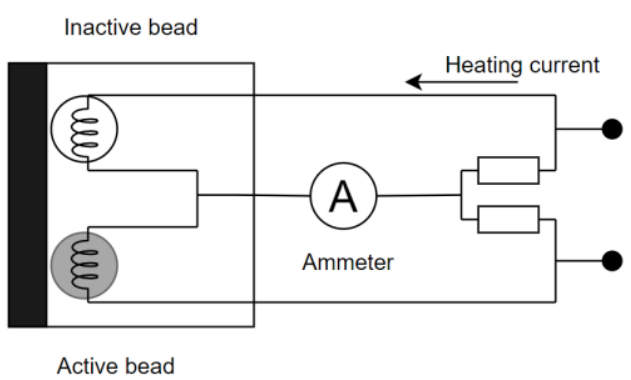

Figure 1. Pellistor (Hübert et al., 2011)

The thermoelectric sensor is although very similar to pellistors, these are based on the Seebeck effect. „If two semiconductors $\mathrm{a}$ and $\mathrm{b}$ are joined together at the hot point and a temperature difference $\Delta \mathrm{T}$ is maintained between this point and the cold point, then an open circuit voltage $\Delta \mathrm{V}$ is developed between the leads at the cold point. This effect, called Seebeck effect after its discoverer T. J. Seebeck." (Van Herwaarden et al., 1986) In a thermoelectric type catalytic sensor there are two cells. The first is the reference cell, which creates a reference point for the measurement cell, by only measuring the carrier gas. In the measurement cell the hydrogen is mixed with the carrier gas. This will create a temperature difference between the two cells depending on the concentration of hydrogen. (Hübert et al., 2011) The thermoelectric sensor is shown in Figure 2.

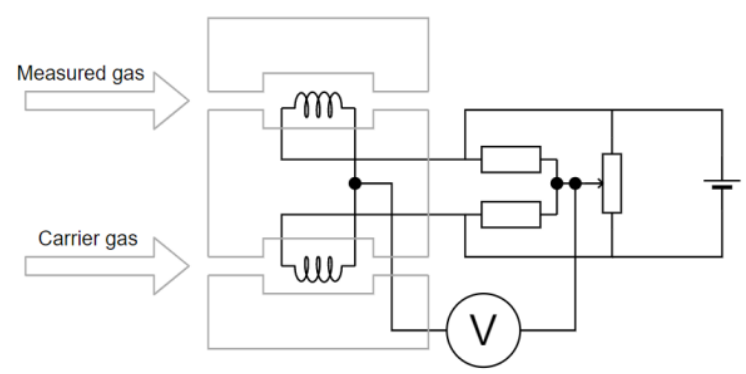

Figure 2. Thermoelectric sensor (Hübert et al., 2011)

The thermal condutivity sensors. The basic principle for thermal conductivity sensors is that we measure the heat loss on a surface to the surrounding gas, which correlates to concentration of hydrogen in the carrier gas. Typically, these sensors can be separated into two groups. The first group has two chambers in which each contain a thermoresistor embedded in a bead. One chamber is closed with a reference gas and the other is open for the measured media. The two beads are connected in with a Wheatstone bridge, so we can measure the change in resistance by the heat loss and thus the concentration of hydrogen. The second type of thermal conductivity sensors does not have reference cham- 
ber. In this structure the sensor has a hot and a cold side and these are connected with the carrier gas. The measured property is the power required to keep the temperature of the hot side on the given value. The rise in power means that the composition of the measured gas has changed and has an element with higher thermal conductivity, so it can relate to the concentration of this element. (Jessop, 1966) The thermal conductivity sensor is shown in Figure 3.

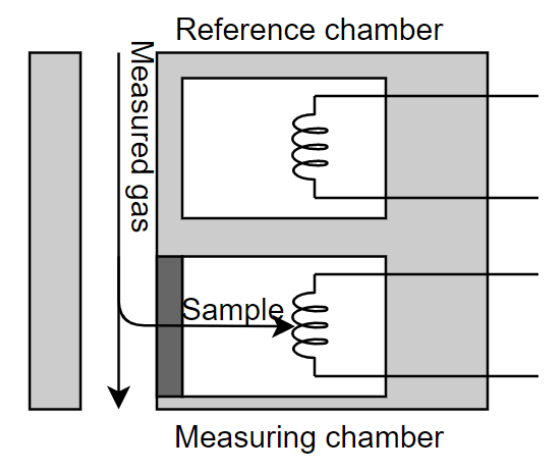

Figure 3. Thermal conductivity sensor (Jessop, 1966)

The electrochemical sensors. This sensors are based on the change in charge in electrical properties due to electrochemical reactions. These kinds of sensors have two electrodes on both ends. These are called a sensing electrode and a counter electrode and made from a noble metal, usually platinum. The electrodes are connected with some kind of electrolyte. The reference electrode has an inlet to the diffusion material, which is covered by a gas permeable material. During operation hydrogen gas diffuses through the gas permeable material and changes electrical properties in the diffusion material. Electrochemical sensors can be amperometric or potentiometric sensors. In amperometric sensors, the diffusion induces an electron flow in between the electrodes, which is proportional to the hydrogen concentration. (Stetter et al., 2008) The potentiometric sensors on the other hand have nearly zero current flow. The measured property is the voltage difference between the electrodes. (Hübert et al., 2011) The electrochemical sensor is shown in Figure 4.

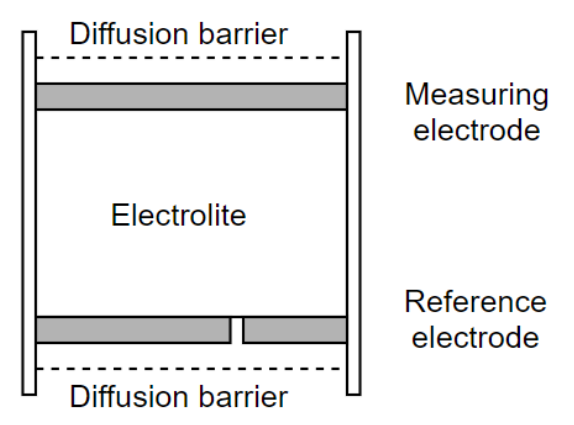

Figure 4. Electrochemical sensor (Stetter et al., 2008)

The resistance based sensors. If metal-oxide is exposed to reducing gases, it changes its electrical properties. The semiconductor metal-oxide sensors use this principle. The metal-oxide layer is applied to an insulating substrate material between two electrodes. When used, the sensor is heated with a 
heater element. If a reducing gas, such as hydrogen is introduced to the sensitive layer over the metaloxide, changes the resistance between the two electrodes. Another type of resistance-based sensors is the metallic resistor. These sensors usually use a thick palladium film. This metal has great potential for hydrogen sensors because it can easily absorb hydrogen. (Hübert et al., 2011) The resistance based sensor is shown in Figure 5.

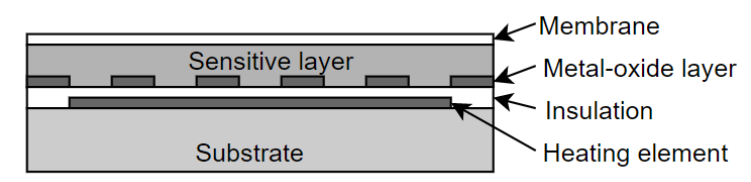

Figure 5. Resistance based sensor (Hübert et al., 2011)

The work function based sensors. "The work function of a metal can be defined as the minimum energy required to extract one electron from a metal." (Hsizl et al., 1979) These sensors usually have a metal, an oxide and a semiconductor layer (MOS). For hydrogen measurements there are three typical structures. The first one consist of a metal and a semiconductor and often referred to as Schottky-type sensors. When the metal (palladium or platinum) absorbs hydrogen, the Schottky barrier height changes by the proportion of the concentration. Another type of sensor called a Metal-insulatorsemiconductor transistor. Similarly to the Schottky type, when the metal layer absorbs hydrogen, the work function changes and a voltage difference can be measured between the source and the drain. The value of the voltage correlates to the hydrogen concentration. The third type of sensor is the metal-insulator-semiconductor capacitor. The structure of the sensor is also very similar to the diode, but the insulating oxide layer is thicker and thus act like a capacitor. When the metal absorbs hydrogen, a change in the C-V plot can be measured. (Hübert et al., 2011) The work function based sensor is shown in Figure 6.

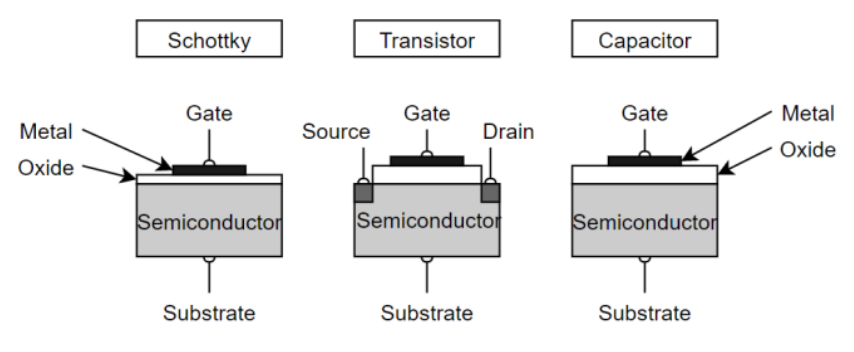

Figure 6. Work function based sensor (Hsizl et al., 1979)

The mechanical sensors. This sensors utilize the expansion of the material when hydrogen is absorbed. In these sensors there are micro machined cantilevers with palladium coating on one side. When hydrogen is introduced to the environment, the palladium absorbs it and starts to expand. The material of the cantilever is chosen to have a much lower permeability than palladium. When the coating starts to expand in a much higher rate, than the cantilever, a tension appears, and the cantilever starts to bend similarly to a bimetal. This then can be measured. (Chou et al., 2007)

The optical sensors. Materials introduced to hydrogen gas also can change optical properties. These sensors usually have a thin palladium layer on top of a titanium layer for adhesion on an optical fibre. This means that with these layers the sensors will be selective to hydrogen. As the optical hydrogen 
sensors consists of similar materials, they mostly differ in measurement principles. A micromirror can be created by placing a thin layer of palladium on the end of an optical fibre. When hydrogen absorbed by this layer, the reflectivity changes. This change can be measured and correlated to concentration. (Hübert et al., 2011) The principle of Surface plasmon resonance can also be used in optical measurements. "Surface plasmon resonance (SPR) technique is an optical method for measuring the refractive index of very thin layers of material adsorbed on a metal. A fraction of the light energy incident at a sharply defined angle can interact with the delocalized electrons in the metal film (plasmon) thus reducing the reflected light intensity." (Pattnaik, 2005) This effect is caused by the absorption of hydrogen in the palladium layer. Etched gratings in the core of the optical fibre coated with palladium also can be used. These are called Fibre Bragg gratings. The expansion of these gratings when it absorbs hydrogen causes the wavelength of the light traveling in the optical fibre and thus can be measured. (Hübert et al., 2011)

The acoustic sensors. The last kind of hydrogen sensor investigated is based on acoustic properties. Piezoelectric quartz crystals used in quartz crystal microbalance sensors use the resonation caused by the deformation of the piezo crystal. Hydrogen absorption can cause this deformation. The resonance frequency can then be measured. (Hübert et al., 2011) Another method is to use surface acoustic waves. "In general, SAW devices generate and detect acoustic waves using interdigital transducers (IDT) on the surface of a piezoelectric crystal. In this way, the acoustic energy is strongly confined at the surface of the device in the range of the acoustic wavelength, regardless of the thickness of the complete substrate. For this reason, the wave is potentially very sensitive towards any change on the surface, such as mass loading, viscosity and conductivity changes." (Länge, 2008) In the case of hydrogen sensors one interdigital creates acoustic waves and another picks them up and convert them electronic signals. A solid material placed between these IDT-s, the change in surface acoustic waves caused by hydrogen can than be measured. (Hübert et al., 2011)

\section{Industrial solutions}

The Hach Orbisphere data acquisition and electrochemical sensor is suitable for measuring oxygen and hydrogen in the EX (explosive) area. The two different sensors are suitable for measuring both liquid and gas samples. The device consists of a measuring data logger and an electrochemical sensor that can be connected to it. The sensor is essentially an electrochemical cell comprising a metal anode and a metal cathode with an electrolyte fluid between them. [10]

The sensor consists of a central (cathode) and an external (anode) electrode, to which a current flows between them by applying a voltage. The electrodes are separated from the test liquid or gas by a gas-permeable membrane. In the case of hydrogen, the two electrodes are arranged in reverse order. In the event that a barrier penetrates the sensor through the membrane, they will dissolve in the electrolyte to change its conductivity. The varying current is proportional to the concentration of the test gas. [10]

The sensor can also be mounted inline up to $60 \mathrm{bar}$, in which case it is connected to a threaded piece welded to a pipeline. This is important because the natural gas network is typically 60 bar. Installation at high pressure is unfortunate because it can cause distortion in the membrane.That is, if the sensor is to be used at high pressure, the calibration must be performed at high pressure so that the hydrogen concentration at the time of calibration is known. In the case of inline measurement, the cycle time of the hydrogen sensor is 6 seconds. 
The sensor is sensitive to the temperature. The sensor contains an electrolyte which freezes around $-5^{\circ} \mathrm{C}$, i.e. it should not go below this in any way, but it is preferable not to cool the gas below $0^{\circ} \mathrm{C}$. If it is not possible to comply with this temperature requirement, installation of the sensor inline is not recommended.

That is, in this case, a flow-through cell installation would be appropriate by draining the flowthrough gas. This type of sample preparation increases the cycle time, the magnitude of which depends on the medium used, but typically no more than 3-5 seconds. In other words, the cycle time of the sensor is no more than 10 seconds. [10]

That is, the sensor is sensitive to pressure and temperature and requires frequent calibration (every six months), but its cycle time is short. Thus, if it is important to determine the hydrogen concentration as soon as possible (e.g. in the case of regulation), it may be an appropriate solution.
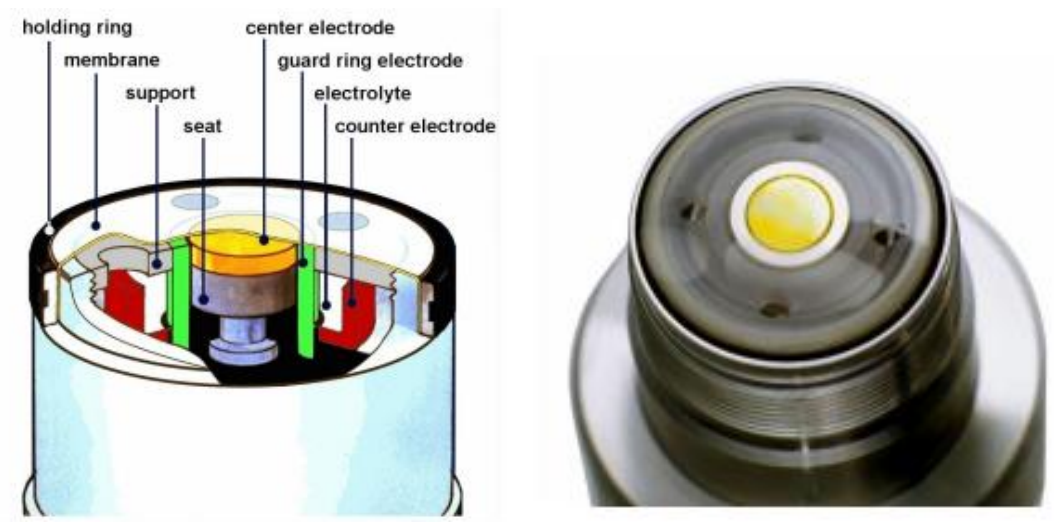

Figure 7. Hach sensor [10]

The Optograf Analyzer is a laser-based analyzer developed for petrochemical and gas treatment purposes. The apparatus generates spectra similar to the chromatograms generated by the chromatographs, which can be analyzed in a similar manner. The Optograph does not include valves, furnaces and does not require carrier gas. This allows the device to operate with low maintenance costs and high reliability.

Probes can be connected to the central unit of the analyzer with fiber optics. Simultaneous measurement of several, up to four data streams is possible, which shortens the cycle time. An AirHead TM gas phase probe can be connected to the analysis unit, which is designed so that gas phase monitoring of chemical processes is possible even at low gas concentrations (100 ppm - gas dependent). The design of the AirHead probe is easy to install, the probe shell is made of stainless steel. The AirHead probe features Kaiser's patented multipass approximation, which improves the analyzer's overall detection limit. The Optograph can test gas mixtures in which $\mathrm{H}_{2}, \mathrm{~N}_{2}, \mathrm{O} 2, \mathrm{CO}, \mathrm{CO}_{2}, \mathrm{H}_{2} \mathrm{~S}, \mathrm{CH}_{4}, \mathrm{C}_{2} \mathrm{H}_{4}$, $\mathrm{C}_{2} \mathrm{H}_{6}, \mathrm{Cl}_{2}, \mathrm{~F}_{2}, \mathrm{HF}, \mathrm{BF}_{3}, \mathrm{SO}_{2}$ are present in concentrations of up to $0.1 \%$. Furthermore, said substances can be tested in concentrations of up to $100 \%$.

There are two main elements to the sample system. The so-called Airhead probe (this includes the optics) and in our case the so-called Opto AST sampling cabinet.

Without pressure reducer approx. the optical measuring head can be used up to a pressure of 71 bar. This probe is connected to the fitting in the OptoAST cabinet.

Although this is not an inline device, it is important to note that it requires much simpler sample preparation compared to a chromatograph because the sensor is directly at the technology site. The 
advantage is that 4 measuring heads can be fitted to an analyzer. One of the disadvantages of the tool is that the total analysis time is typically 2-4 minutes based on the quote, which includes the measurement cycle time and the sample preparation time. Another disadvantage is that it does not measure accurately below $1.5-2 \%$. (Optograf)

\section{Summary}

Green hydrogen combustion processes only produce water vapor, but not carbon dioxide. An innovative use of hydrogen to mix with natural gas to deliver the mixture to end users using existing natural gas storage and networks, thereby achieving increased performance.

However, when using the mixture, it is necessary for the natural gas storage technology to be equipped with hydrogen concentration transmitters. The paper is a review in which hydrogen sensors and two industrial solutions are presented.

In the future, it would be important to examine the efficiency and speed of each hydrogen meter and to categorize them based on their use.

\section{Acknowledgement}

This research was carried out in the framework of „Aquamarine Project - Hydrogen energy storage innovation at Hungarian Natural Gas Storage Ltd.", Call for Proposals No. 2020-3.1.2-ZFR-KVG2020-00001"

\section{References}

[1] Hübert, T., Boon-Brett, L., Black, G., Banach, U. (2011). Hydrogen sensors - A review. Sensors and Actuators B: Chemical, 157(2), 329-352. https://doi.org/10.1016/j.snb.2011.04.070

[2] World Nuclear Association. (2021). Heat values of various fuels. https://worldnuclear.org/information-library/facts-and-figures/heat-values-of-various-fuels.aspx

[3] Van Herwaarden, A. W., Sarro, P. M. (1986). Thermal Sensors Based On The Seebeck Effect. Sensors and Actuators, 10(3-4), 321-346. https://doi.org/10.1016/0250-6874(86)80053-1

[4] Jessop, G. (1966). Katharometers. Journal of Scientific Instruments, 43(11), 777-782. https://doi.org/10.1088/0950-7671/43/11/301

[5] Stetter, J. R., Li, J. (2008). Amperometric gas sensors - A review. Chem. Rev., 108(2), 352366. https://doi.org/10.1021/cr0681039

[6] Hsizl, J., Schulte F. K. (1979). Work function of metals solid surface physics. Springer Tracts in Modern Physics, 85, ISBN 978-3-540-09266-7. Springer-Verlag, p. 1

[7] Chou, Y-I., Chiang, H-C., Wang, C-C. (2007). Study on Pd functionalization of microcantilever for hydrogen detection promotion. Environmental Engineering, 10(2)

[8] Pattnaik, P. (2005). Surface plasmon resonance. Appl Biochem Biotechnol., 126(2), 79-92. https://doi.org/10.1385/ABAB:126:2:079

[9] Länge, K., Rapp, B. E., Rapp, M. (2008). Surface acoustic wave biosensors: a review. Anal Bioanal Chem., 391(5), 1509-19. https://doi.org/10.1007/s00216-008-1911-5

[10] Hach - ORBISPHERE Model 31xxx Electrochemical Sensors USER MANUAL 06/2018, Edition 10

[11] Optograf Analyzer Laser-Based „Optical” Chromatograph 